Behavioral and Brain Sciences

http://journals.cambridge.org/BBS

Additional services for Behavioral and Brain Sciences:

Email alerts: $\underline{\text { Click here }}$

Subscriptions: Click here

Commercial reprints: Click here

Terms of use : Click here

\title{
Plasticity: Implications for opioid and other pharmacological interventions in specific pain states
}

Anthony $\mathrm{H}$. Dickenson

Behavioral and Brain Sciences / Volume 20 / Issue 03 / September 1997, pp 392 - 403

DOI: null, Published online: 08 September 2000

Link to this article: http://journals.cambridge.org/abstract S0140525X97241488

How to cite this article:

Anthony H. Dickenson (1997). Plasticity: Implications for opioid and other pharmacological interventions in specific pain states. Behavioral and Brain Sciences,20, pp 392-403

Request Permissions : $\underline{\text { Click here }}$ 


\title{
Plasticity: Implications for opioid and other pharmacological interventions in specific pain states
}

\author{
Anthony H. Dickenson \\ Department of Pharmacology, University College London, \\ London WC1E 6BT, United Kingdom \\ Electronic mail: anthony.dickenson@ucl.ac.uk
}

\begin{abstract}
The spinal mechanisms of action of opioids under normal conditions are reasonably well understood. The spinal effects of opioids can be enhanced or reduced depending on pathology and activity in other segmental and nonsegmental pathways. This plasticity will be considered in relation to the control of different pain states using opioids. The complex and contradictory findings on the supraspinal actions of opioids are explicable in terms of heterogeneous descending pathways to different spinal targets using multiple transmitters and receptors - therefore opioids can both increase and decrease activity in descending pathways. These pathways could exhibit considerable plasticity. There is increasing evidence that delta opioid receptor agonists have the potential to replace morphine as major analgesics with reduced side-effect profiles. The concept of preemptive analgesia, based on preventing the induction of some of the negative plastic influences on opioid controls and the detrimental effects of pain, is sound, but experimental verification in the clinical setting is difficult. For example, a delayed compensatory upregulation of inhibitory systems, particularly in inflammation, may counter persistent painful inputs. Combination therapy with opioids may be beneficial in many pain states where either negative influences are blocked or inhibitory controls are enhanced. Finally, developmental aspects of these systems are discussed in connection with the treatment of pain in young children, where inhibitory systems in the spinal cord are immature.
\end{abstract}

Keywords: analgesia; cholecystokinin; development of pain; excitatory amino acids; hypersensitivity; nociception; opioids; peptides; spinal cord

\section{Introduction: Plasticity in pain}

The two major types of clinical pain arise from distinct events in the periphery. Inflammatory pain arises from tissue damage such as that produced by trauma, surgery, childbirth, or invasion of tissue by a tumour. The second type of pain is termed "neuropathic" pain and results from damage to a nerve; trauma, surgery, and cancer can also cause this type of pain. In the case of inflammatory pain, the damage to tissue causes the local production of a number of chemical mediators that sensitize and/or activate the peripheral endings of nociceptive C-fibres (Dray et al. 1994). With neuropathic pain, however, activity is set up in the nerve itself. Regardless of the origin of the pain, impulses are conveyed to the first synapse in the dorsal horn of the spinal cord, where the interplay between excitatory and inhibitory events determines the ascending messages that are transmitted to higher centres. However, descending controls from the brain stem can be triggered and further alter processing in the spinal cord (Besson \& Chaouch 1987; Fields et al. 1988). Opioid analgesics can exert controls on these events by direct actions on the spinal cord but can also interact with systems at the origins of the descending controls (Duggan \& North 1984; Dickenson 1994a). Thus, at a number of levels there is the potential for alteration in the messages that ultimately give rise to the final sensation of pain. This article is an attempt to bring together some of the interactions between excitatory and inhibitory events to explain some of the different characteristics of inflammatory and neuropathic pain and to investigate how alterations in these systems can give rise to difficulties in treating certain pains, especially neuropathic pains.

It is well established that the repertoire of the adult central nervous system (CNS) is not fixed and immutable. Plasticity, the ability of central nervous function to change in response to internal and external events, can be due to alterations in connectivity (Woolf \& Doubell 1994; Dray et al. 1994). Plasticity is of great relevance to the control of these different pain states (McQuay \& Dickenson 1990), yet plasticity can also result from a relatively rapid induction and activation of different pharmacological systems under different circumstances (Dickenson 1994a; 1994b; McMahon et al. 1993; Price et al. 1994b; Woolf 1994). This target article will concentrate on interactions between spinal pharmacological systems and opioid analgesia. States of increased pain transmission, central hypersensitive states (Woolf 1983), can result from activation of spinal systems that do not participate in the responses to brief stimuli. In these cases where the level of excitatory transmission is augmented, opioid inhibitions will need to be increased to compensate. Consequently, the actions of opioids are not fixed but highly dependent on activity in other transmitter systems that in turn appear to be influenced by different types of pain. This target article will consider this plasticity in relation to the control of different pain states using 
opioids as a theme. The main emphasis will be on results from models of acute and more prolonged pains such as that arising from inflammation and neuropathy and I will attempt to discuss why the actions of opioids may differ in different pain states. Thus, pain arising from tissue damage (inflammatory nociceptive pain) can respond well to opioids whereas neuropathic pain (arising from peripheral or central nerve damage) and allodynia (where touch is perceived as pain) can show poor opioid sensitivity.

\section{Opioid receptors}

Opioids act by activating three opioid receptors: the mu, the delta, and the kappa (Kosterlitz 1985). Whereas the endogenous opioid peptides, the natural ligands for the receptors - namely the enkephalins, dynorphins, and endorphin family - are not entirely specific for any one of these receptors, a number of synthetic agents with high selectivity are available to study the individual receptors (Dickenson 1994a; Kosterlitz 1985). Neurones producing the different opioids can be unequivocally identified now using messenger RNA probes for the precursor propeptides. Opioid peptide synthesis can be altered in animal models of different pain states, for example, dynorphin levels in the spinal cord during inflammation are increased enormously due to the switching on of the gene for the synthesis of the parent propeptide (Dubner \& Ruda 1992). The biological lifetimes of the endogenous opioids, particularly the enkephalins, are brief due to rapid peptidase degradation. It is now possible to protect the enkephalins from breakdown by the use of peptidase inhibitors, some of which are now active by systemic routes (Roques et al. 1993). The use of these agents together with the synthesis of stable analogues of the endogenous opioids themselves has provided the means for the study of the roles and function of opioids and their receptors with far greater selectivity than with only the endogenous opioids.

2.1. Mechanisms of opioid analgesia. There are three key mechanisms of action for opioids. The underlying events by which opioids interfere with the transmission of pain are the same as the mechanisms by which opioids cause their other actions, including side effects (Dickenson 1994a; Duggan \& North 1984). These are:

1. A presynaptic action on the terminals of neurones whereby transmitter release is reduced by activation of opioid receptors. In tissues where relative receptor location has been gauged, the number of presynaptic opioid sites predominates over postsynaptic locations.

2. There are significant numbers of postsynaptic opioid receptors and after activation the resultant hyperpolarisation reduces evoked activity in the neuronal pathways. The postsynaptic effects can be on cell bodies of output neurones, interneurones, or dendrites.

3. An alternative postsynaptic action involves disinhibition: in a circuit of two neurones, where the second cell is held in check by an inhibitory neurone, opioid inhibition of the first neurone allows the second cell to become active.

These actions produce analgesia at a number of sites in the nervous system. The two key sites would appear to be a spinal and a midbrain/brain stem action in normal circumstances, but an additional peripheral site in inflamed tissue can also be induced.

\section{Spinal analgesia}

Opioid receptors in the spinal cord are a critical site in the production of analgesia. Spinal opioid analgesia demonstrates how basic research in animals can have a rapid and important application to the clinical relief of pain. Opioid inhibition of nociceptive neurones in spinal animals and then evidence for analgesia following epidural and intrathecal opioids in animals was soon followed by clinical usage (Besson \& Chaouch 1987; Yaksh \& Nouiehed 1985).

3.1. Presynaptic actions. The highest levels of opioid receptors in the spinal cord are around the $\mathrm{C}$-fibre terminal zones in lamina 1 and the substantia gelatinosa with lower levels found in deeper layers. The best current estimates suggest that the mu receptor forms $70 \%$, the delta $24 \%$, and the kappa $6 \%$ of the total opioid sites in the rat spinal cord (Besse et al. 1990). The idea that kappa levels are higher in the mouse and guinea pig spinal cord has been put forward. However, studies in species other than the rat have not been carried out with the most selective ligands for the receptors and so may not represent the true relative distribution of the receptors. We lack systematic quantitative studies in a number of species on the relative distribution of the receptors at a variety of CNS sites. Recent studies using probes for the selected sequences of the delta receptor have shown unequivocally that many of these receptors are located presynaptically on afferents and in close apposition to enkephalin-containing cells (Dado et al. 1993). In addition, spinal application of antisense to the delta receptor has shown that this leads to a marked reduction in deltamediated analgesia without alteration of the effects of morphine (Uhl et al. 1994).

The relative numbers of presynaptic and postsynaptic receptors can be calculated after nerve section and the former predominate. The proportions of presynaptic opioid receptors in the spinal cord varies from $70 \%$ to $50 \%$, with over $70 \%$ of the total mu receptor sites (Besse et al. 1990), along with large numbers of delta receptors (Dado et al. 1993), on the afferent terminals. Given the large number of receptors, it is not surprising that evidence for a presynaptic action of opioids emerges from studies of opioid inhibition of C-fibre evoked release of transmitters (substance P and glutamate) as well as in vitro and in vivo electrophysiological studies (Dickenson 1994a; Yaksh \& Malmberg 1994; Yaksh \& Nouiehed 1985). However, other approaches have failed to demonstrate this presynaptic action (e.g., Lang et al. 1991).

Presynaptic actions on transmitter release result from an opening of potassium channels ( $\mathrm{mu}$ and delta receptors) or a closing of calcium channels (kappa), both of which lead to a reduction in calcium influx into C-fibre terminals - thus diminishing transmitter release (North 1989). C-fibres are believed to release a number of coexisting transmitters including the tachykinins, excitatory amino acids, and a number of excitatory peptides that act on multiple receptors (Besson \& Chaouch 1987; Dickenson 1994a; Dray et al. 1994). Consequently, the presynaptic action of opioid's ability to reduce the release of many transmitters will be a highly effective route to analgesia, since it will be equivalent to the block of multiple postsynaptic receptors. It is not probable, therefore, that any single antagonist of one of these postsynaptic receptors will have sufficient efficacy to compete with the opioids as do powerful analgesics in acute and chronic pains. An exception to this is likely to be agents 
acting directly, or indirectly, to modulate the N-methylD-aspartate (NMDA) receptor in pains where central hypersensitivity, an augmented spinal response to a low or moderate afferent input, is generated.

Section of a peripheral nerve will lead to degeneration of the nerve and the presynaptic receptors synthesized in the dorsal root ganglion will be lost (reducing mu opioid receptor sites in the dorsal horn by 70\%; see Besse et al. 1990). It would be beneficial to know if less severe nerve damage impairs the production of functional opioid receptors. However, in animal models where the presynaptic opioid receptors have been removed by prior rhizotomy, whether electrophysiological with systemic dosing in spinal preparations (Lombard \& Besson 1989) or behavioural with spinal application (Xu \& Wiesenfeld-Hallin 1991), morphine is still effective, although higher doses are required in the former case. Thus when there is a loss of opioid receptors, there will be a reduction in opioid actions that should predictably be overcome by dose escalation (Lombard \& Besson 1989). If nerve damage does lead to a loss of some of the spinal opioid receptors, one could predict that opioid delivery to supraspinal sites would target the normal population of supraspinal receptors. In clinical practice there is a reduced systemic opioid effectiveness in neuropathic pains (Arner \& Meyerson 1988) that can be overcome by increasing the dose in some circumstances (Jadad et al. 1992; Portenoy et al. 1990). Dose increases may not always be possible, since side effects may become intolerable. When the side effects can be controlled and increase in dose does not overcome the pain, we have to suspect that opioid receptor loss or dysfunction is not the only factor.

3.2. Postsynaptic actions. Evidence for functional opioid actions at postsynaptic receptors is based on electrophysiological and behavioural approaches (Duggan \& North 1984; Lombard \& Besson 1989). Postsynaptic hyperpolarisations again result via the opening of $\mathrm{K}^{+}$channels or the closing of calcium channels (North 1989). These receptors could hyperpolarise the dendrites of projection neurones and interneurones (both would be selective for noxious transmission) as well as the cell body of projection cells that may not be selective for nociceptive inputs, since many but not all neurones in the dorsal horn receive both nociceptive and tactile inputs.

An important indirect postsynaptic action is the opioid disinhibitory effect mediated via GABA and enkephalin neurones in the substantia gelatinosa, which in turn leads to an inhibition of output neurones. Thus in this instance, neurones can be recorded in the substantia gelatinosa that are facilitated by opioids, an action that requires $\mathrm{GABA}_{\mathrm{A}}$ receptor function. There is both morphological and electrophysiological evidence to support this action (see Magnuson \& Dickenson 1991).

These postsynaptic actions of opioids present some problems of interpretation, since any direct hyperpolarisation of a cell soma would inhibit all responses of the cell including the innocuous inputs onto convergent or multireceptive cells. However, many of the opioid receptors in the substantia gelatinosa could be on the dendrites of the deep cells penetrating into the C-fibre terminal zone; inhibitory effects here would also be selective, as they are likely to be spatially distinct from the large fibre inputs. Another possibility is that the postsynaptic disinhibitory effects of opioids selectively feed onto nociceptive circuitry. When allodynia and some of the hyperalgesias are transmitted through A-fibre afferents, a poor sensitivity to opioids might be found (Yaksh 1989), since the only opioid control of A-fibre inputs is via the relatively small number of postsynaptic receptors on the output neurones (Besson \& Chaouch 1987; Duggan \& North 1984). Doses of morphine that abolish C-fibre-evoked responses in normal animals have only minor effects on A-fibre activity (Dickenson \& Sullivan 1986). In these pain states, novel nonopioid therapy directed at the spinal systems generating the tactile-evoked pain may have to be considered (Yaksh 1989).

\section{Alternatives to morphine?}

Surprisingly, it is unclear whether different opioids may have slightly different ranges of pharmacological actions that could allow a choice of opioid for different pains. Clinical studies comparing different opioids in different pain states are needed. Morphine at physiological doses probably acts only at the mu receptor. This drug has a high affinity for the mu receptor, a relative affinity of 50 times less for the delta receptor, and a minimal affinity for the kappa receptor (Kosterlitz 1985). Thus relatively nonselective effects could occur with very high doses such as those achieved both in neurochemical and in binding studies where non-mu effects of morphine have been reported. However, in vivo, doses of spinal morphine, which are sufficient to abolish the C-fibre-evoked responses of dorsal horn nociceptive neurones, are probably entirely mu receptor mediated. In support of this, there is no evidence for mu-delta cross tolerance from physiological studies (Kalso et al. 1993). Since morphine is the standard opioid for clinical practice, plasticity that is related to $\mathrm{mu}$ receptor mechanisms is of great importance (Dickenson 1994a).

Possible opioid drugs that act on receptors other than the $\mathrm{mu}$ receptor for morphine are analgesics with reduced morphine-like side-effect profiles. As is the case with any transmitter system, the greater the number of receptors the greater the chance that the desirable effects can be separated from the unwanted effects. With opioid receptors, a further division of the receptors from the main three - the mu, delta, and kappa - has been proposed. The mu receptor has been suggested to consist of a mu 1 and a mu 2 subtype (Pasternak \& Wood 1986); the delta has been also subdivided and the kappa receptor has been divided into three subtypes ( Jiang et al. 1991; Traynor 1989). Whether these subtypes have functional consequences remains to be seen: physiological consequences are not yet discerned except for the delta subtypes where there is evidence for differential effects of the two receptors (Jiang et al. 1991). The recent cloning of the opioid receptors (Uhl et al. 1994) will further facilitate this task, because probes based on the receptor sequence will provide unequivocal proof of location of the particular receptor and important insights into the mechanisms of opioid actions and the existence of subtypes. At the present time there is no evidence from the cloning studies for receptor subtypes: the receptors that have been isolated, whether mu, delta or kappa, were single identical species. There may be alternative splicing that produces the subtypes or local neuronal tissue environments that allow the subtypes to be expressed and, in addition, the cell lines used so far may underestimate the variability within the opioid receptor family (Uhl et al. 1994). However, it is known that the rat and mouse opioid 
receptors are, as far as can be determined, identical in structure and pharmacology to the human receptors, further verifying the important links between animal studies and clinical practice (Uhl et al. 1994).

4.1. Delta opioids. Antagonists for the opioid receptors have demonstrated the independence of mu, delta, and kappa receptors in terms of antinociception, although there have been problems demonstrating kappa receptor agonist effects in some studies (Millan 1990). The independent analgesic effects following activation of non-mu receptors indicate potential for opioid analgesics that are delta or kappa agonists. Kappa opioids are not always particularly effective analgesics in animals and this appears to be reflected in the initial early clinical studies with these drugs in humans. The delta receptor may well be an important target for novel opioid therapy. Animal studies have shown that opioids selective for the delta receptor can equal the analgesic effects of morphine by actions at both spinal and supraspinal sites in a number of nociceptive tests (Dickenson et al. 1987; Jiang et al. 1991; Sullivan et al. 1989a). One could also predict reduced respiratory depression and gastrointestinal effects of delta as compared to mu opioids. There are now reports of potent and selective nonpeptide delta opioids, which have been tested in a number of paradigms in animals. A highly selective delta opioid, SNC 80 , has been produced. It was found to be effective by central and systemic (including oral administration) routes, and analgesic effects were reversed by a number of delta but not mu opioid receptor antagonists. Importantly, in tests of respiratory function, SNC 80 stimulated rather than reduced respiratory rate in sheep (Bilsky et al. 1995). The potential of delta opioids is therefore high and eventually delta opioids may be clinical alternatives to morphine. As will be discussed in sections 5 and 7.1, there are suggestions that some of the reduced effects of morphine in neuropathy may be due to negative effects of cholecystokinin on mu receptors - these effects do not extend to delta-mediated actions. Consequently, it is possible that delta opioids may be better analgesics than mu opioids in the treatment of neuropathic pains.

4.2. Endogenous opioids. What about endogenous opioids? The enkephalins are rapidly degraded by membrane-bound peptidases. The synthesis of peptidase inhibitors has been a successful strategy, so that kelatorphan, a mixed peptidase inhibitor inhibiting at least two of the important breakdown enzymes, affords almost complete protection to the enkephalins (Roques et al. 1993). The spinal application of the inhibitor produces a reduction of nociceptive responses of cells, with the pool of enkephalins protected by the inhibitor likely to be derived from both a segmental release and from descending pathways activated by the stimulus. The inhibitions are reversed by a selective delta antagonist (Dickenson et al. 1987a). The very recent reports of RB 101, a systemically active mixed peptidase inhibitor, constitutes the next stage toward the clinical application of this novel approach to pain relief. In addition, the side-effect profile of RB 101 appears to be unlike that of morphine in terms of physical and psychological dependence (Roques et al. 1993).

4.3. Novel peripheral analgesia. Whereas opioids lack peripheral actions in undamaged tissue, there is now good evidence that the consequences of inflammation can reveal a novel site of opioid action that appears rapidly (Stein 1994). The synthesis of opioid receptors occurs in dorsal root ganglion cells as well as other locations in the body. These receptors are transported in the fine afferent fibres in both directions; the centrally directed receptors become the presynaptic receptors and the peripherally transported receptors somehow become active only following inflammation. The relative effectiveness of mu, delta, and kappa receptor activation to elicit peripheral analgesia varies between models, but in arthritic states all three are active (Stein et al. 1989). Alongside the appearance of the functional opioid receptors on afferent nerves, the arrival of endogenous opioid peptides at the injury site seems to be related to immune cell proliferation. Thus opioids unable to penetrate the CNS and, as a result, devoid of central side effects may be good analgesics in inflammatory states via these peripheral sites. There have now been a number of clinical studies on this effect, the bulk of which have been positive. Thus the local application of morphine into the knee joint in patients has been shown to produce a local analgesic effect. A recent study has also shown that the degree of analgesia can be related to the amount of tissue damage and, presumably, to the degree of inflammation (Stein 1994). Peripherally acting opioids may then have potential analgesic effects in inflammation (Stein 1994; Stein et al. 1989).

4.4. Supraspinal analgesia. The first demonstration of opioid actions within the CNS consisted of analgesia seen following intraventricular morphine. Numerous supraspinal sites of opioid analgesia have been established (Besson $\&$ Chaouch 1987). These have now been localized to areas in the medial brain stem around the nucleus raphe magnus and extending rostrally to periaqueductal and periventricular grey and other areas with the monoamines appearing to be critical transmitters in these pathways (Yaksh et al. 1988). The roles of these areas in morphine analgesia have been based on microinjection studies and the ability of naloxone, when applied locally into these areas, to reduce the effects of systemic morphine.

The mechanisms of action in opioids at these supraspinal levels still is unclear, particularly about how they interact with descending inhibitory controls. Whereas opioid induced increases and decreases in descending inhibitory controls have been reported, the roles of these descending pathways in different models of various pain states are unknown. We need more information on the physiological and pharmacological bases for supraspinal analgesia in animal models of persistent pain in order to form a basis for the potential use of manipulation of the monoamines in difficult clinical pains.

Examination of the anatomy and the pharmacology of the descending systems may provide a basis for these disparate results with regard to opioid interactions with descending controls. First, descending controls originate from many different areas of the brain stem and midbrain and, in addition, a complex pharmacology exists in these descending pathways (Yaksh et al. 1988). Noradrenaline, 5HT, enkephalin, and substance $P$ are involved, all coexisting in some neurones projecting from the brain stem and midbrain to the spinal cord. In addition to interactions between these transmitters, there are a number of local transmitter systems (cholinergic, GABAergic, and opioid) in the nuclei where the descending controls originate. Direct opioid inhibitions 
or indirect disinhibitions could result from local opioid actions in these supraspinal areas. These opposite effects could themselves be on inhibitory and/or excitatory pathways. A further complexity is that the monoamines have a number of receptors at the spinal terminal sites that when activated could inhibit or excite depending on the receptor an additional complication is the presence of autoreceptor or heteroreceptor control of the release of transmitter at these terminal sites. Whether the postsynaptic receptors are on excitatory or inhibitory elements is also important. Consequently the problem is not to discern the direction of effect of opioids on these systems but to understand the physiological roles and consequences of the mixed opioid actions on these multiple pathways (Dickenson 1994a).

Consideration of the direction of effect of the spinal monoamine receptors serves to illustrate these points. In the case of noradrenaline, there is a general consensus that, notwithstanding a possible role of the alpha-1 receptors, the predominant spinal targets for the transmitter are alpha-2 receptors located in the spinal cord, postsynaptically to the noradrenergic terminals. In a similar manner to the opioid receptors and the afferent nociceptive fibres, these receptors are located both presynaptically and postsynaptically on spinal sensory circuits; there is ample evidence for alpha-2 agonists being effective analgesics in a number of animal models of acute and more persistent pains. In addition, there is little doubt that alpha- 2 agonists synergize with morphine, probably as a result of dual activation of separate receptors with similar locations and effector mechanisms (Dickenson \& Sullivan 1993; Yaksh \& Malmberg 1994). Relatively little is known about the driving force behind pain-related changes in noradrenergic activity in these models. An exception is a report of increased alpha-2 mediated activity in inflammation, but it does not contribute to enhanced spinal opioid effectiveness (Stanfa \& Dickenson 1994a). There may, however, be a supraspinal site of action of noradrenaline in enhancing opioid actions (Hylden et al. 1991).

The effectiveness of tricyclic antidepressants (TADS) for pain relief in humans possibly relates to enhancement of the availability of noradrenaline and serotonin. This is where the problems arise. The number of receptors for serotonin or 5-hydroxytryptamine (5HT) increases on a regular basis. Presently, there are at least seven major receptors with over 20 subtypes. The receptor that underlies descending antinociception at the spinal level is unknown. Thus, increases in 5HT levels in the cord will activate all the receptors irrespective of whether they are excitatory or inhibitory. Knowledge of the particular roles of the monoamine receptors may potentially lead to better therapeutic efficacy by agents (or combinations thereof) acting on particular receptors rather than the indirect indiscriminate activation of multiple receptors produced by the TADS (Max 1994).

Bearing in mind these complexities, how do opioids interact with descending pathways? As many of the sites of opioid actions at supraspinal sites overlap with areas where descending inhibitory controls originate, the simplest situation is that supraspinal opioids increase these descending monoamine inhibitions; in turn these block spinal pain transmission by actions at inhibitory spinal receptors. For opioids to increase descending inhibitions, the mechanism will have to be via disinhibitions (Fields et al. 1988). The clearest demonstration of supraspinal descending inhibi- tory controls that are increased by morphine is that the spinal induction of c-fos, used as a marker of noxious evoked activity, was found to be very clearly reduced by intraventricular morphine (Gogas et al. 1991).

There are other studies, however, that do not find this direction of effect. Diffuse noxious inhibitory controls (DNICs) are descending controls induced by heterosegmental noxious stimulation and partly involve both opioid and serotoninergic mechanisms. Morphine, either given directly into supraspinal tissues or at low systemic doses without direct spinal actions, reduces these descending controls (Le Bars \& Villaneuva 1988). There are considerable difficulties, therefore, in arriving at a simple consensus as to the direction of effect of opioids on descending control systems. However, as discussed above, the multiplicity of these descending controls in terms of their anatomy, their pharmacology, and their spinal projections form a framework in which the various directions of effect of opioids can be incorporated. There is no doubt that whatever the mechanism, supraspinal opioids produce behavioural analgesia (Besson \& Chaouch 1987; Yaksh et al. 1988).

It is highly likely that both the level of pain transmission and the effectiveness of opioids in different pain states is determined by alterations in descending control pathways. Other than studies on alpha-2 adrenoceptors, we are ignorant of the extent of plasticity in these systems.

\section{Plasticity in opioid controls}

Using the previously articulated framework of opioid effects and mechanisms on which to consider plasticity in opioid systems, it is pertinent to consider in this section particular pain states where there is evidence for changed opioid effectiveness. Why are opioids sometimes poorly effective in neuropathic pain states in man and animals (Arner \& Meyerson 1988; Jadad et al. 1992; Portenoy et al. 1990)? Yet there is good evidence that in a number of inflammatory models opioids are more effective than in normal animals (Dickenson 1994a). In addition, as there are transmitter systems in the CNS that can reduce opioid effectiveness, preemptive analgesia should stop the induction of these systems and so provide better pain relief: Why has it been so difficult to provide clear and marked clinical benefits for this approach (McQuay 1994)?

The analgesic effects of morphine can vary in different pain states. The mechanisms behind these changes have been elusive, but their identification and eventual manipulation may be of considerable clinical benefit. First, let us consider the pharmacological systems that can interfere with opioid effectiveness, bearing in mind that pathology can also play a role in nerve section and the loss in number of presynaptically located opioid receptors. There appear to be four major pharmacological factors:

1. Interference with mu receptor function by the metabolite of morphine, morphine-3-glucuronide (M3G). This has been proposed on the basis of behavioural studies but is not supported by electrophysiological and clinical studies.

2. Changes in the levels of the nonopioid peptides, FLFQPQRFamide and/or cholecystokinin (CCK), either spinally where there is very strong evidence for CCK as a regulator of morphine analgesia, or supraspinally as a more global negative influence on opioid actions. 
3. Increased levels of the opioid peptide, dynorphin, which has been shown to occur after persistent pain. In theory this peptide can reduce mu opioid analgesia, but the physiological role of dynorphin as an opioid modulator is not good.

4. An excess of excitatory activity, so that a spinally generated hypersensitive state is induced, against which opioid controls are insufficiently efficacious. The $\mathrm{N}$-methyl-D-aspartate (NMDA) receptor is a very strong candidate for the final common path for generation of this state, and there is poor opioid sensitivity to a number of electrophysiological and behavioural measures of pain where NMDA receptor activation has been induced.

These four possibilities are not mutually exclusive (Dickenson 1994a). Thus, in a particular pain state where opioids are used to treat the pain, NMDA mediation of spinal transmission may be occurring at the same time as elevated spinal CCK and dynorphin levels with high plasma M3G levels (Dickenson 1991). The evidence for and against these systems altering opioid analgesia will be considered in turn.

5.1. Morphine metabolites. The actions of morphine do not end with metabolism. It is now well established that the glucuronidation of morphine produces two major metabolites, morphine-3-glucuronide and morphine-6-glucuronide, each with remarkably different actions. The 6 -glucuronide is more potent than morphine itself, and although the degree of this enhanced action is variable from study to study, it is at least 10 to 30 times more effective in tests of analgesia (Sullivan et al. 1989b). The reasons for this are not obvious; the affinity of morphine-6-glucuronide for the mu receptor is not appreciably greater than morphine itself, although it has more delta and less kappa affinity. However, as discussed earlier with morphine, it is likely that predominant $\mathrm{mu}$ activity underlies the analgesia with morphine-like opioids at therapeutic doses. Yet the other metabolite, M3G (morphine-3-glucuronide), has no affinity for the mu receptor and being unable to bind to the receptor has no opioid actions. Results from behaviour after administration of M3G have nevertheless led to the suggestion that M $3 \mathrm{G}$ is a factor that contributes to reduced opioid sensitivity (Gong et al. 1992; Smith et al. 1990). The metabolite given by the intraventricular route caused marked behavioural agitation that interfered with the tests. In contrast to these studies, there is electrophysiological (where nonspecific effects are less likely to interfere with the results) and behavioural evidence that even with dose ratios of 100:1 (metabolite to morphine), M3G has absolutely no effect on the spinal antinociceptive effects of morphine (Hewett et al. 1993). It is highly unlikely that M3G is an important factor in cases of opioid poorly responsive pain, since (1) the spinal site of action of morphine is a major contributor to systemic analgesia, (2) M3G does not bind to opiate receptors, and (3) in renal insufficiency, where the metabolite will accumulate, opiate effects tend to be enhanced. Thus M3G should not, at present, be used as an excuse not to persevere with or increase the dose of morphine in pain states where opioid responsivity is poor. Patient-controlled analgesia has revealed that neuropathic pain patients can gain relief with morphine - although within this patient group pain control is not as good as in patients with nociceptive pains ( Jadad et al. 1992). Dose escalation can also be effective (Portenoy et al. 1990).
5.2. Antiopioid peptides. Among the numerous factors influencing morphine analgesia, accumulating evidence indicates that the nonopioid peptide cholecystokinin (CCK) is an important physiological modulator of analgesic mechanisms. The exogenous spinal application of CCK and another peptide, FLFQPQRFamide, both nonopioid peptides found within intrinsic neurones in the spinal cord, will prevent mu- but not delta-mediated neuronal inhibitions (Baber et al. 1989; Dickenson 1994a), and both reduce intrathecal morphine analgesia in behavioural studies (Xu et al. 1993). Thus in situations where there is a release of these peptides one would expect a reduction in morphine effects without requiring any change in opioid receptor number. In fact, the ability of these peptides to interfere with analgesia is not restricted to the effects of opioids but also includes alpha-adrenoceptor agonist actions.

CCK has been shown to reduce the analgesic effects of morphine at a number of CNS sites and has also been implicated in the development of opioid tolerance (Baber et al. 1989). Spinal and supraspinal delta opioid mediated analgesias are not altered by CCK and, as a result, if there are physiological situations where CCK reduces mu opioid actions, future clinical delta agonists could be effective.

One of the key sites for these interactions is the spinal cord. Negative results with cDNA probes within dorsal root ganglia in the normal rat make it very unlikely that genuine CCK is found in nociceptive C-fibres in normal animals. Surprisingly, induction of the peptide in afferents occurs after pathological damage to the afferents. The consequences of this with regard to neuropathic pain (Xu et al. 1993) are discussed later in this section. Endogenous CCK in the dorsal horn under nonpathological conditions is thought to originate from both intrinsic neurones found in superficial laminae and descending fibres. The receptors are found both presynaptically (approximately 50\%-60\%) and postsynaptically to the primary afferent fibres, mirroring the mu opiate receptor distribution in the rat spinal cord. The postsynaptic CCK receptors are mainly of the $\mathrm{CCK}_{\mathrm{B}}$ type in the rat spinal cord but of the A-type in the primate, whereas the presynaptic receptors are of the $\mathrm{CCK}_{\mathrm{B}}$ type in all species. (Ghilardi et al. 1992). Thus $\mathrm{CCK}_{\mathrm{B}}$ receptor antagonists will be critical in testing whether CCK influences morphine analgesia in humans (Stanfa et al. 1994).

The mechanism by which CCK attenuates the antinociceptive effect of morphine is not on opioid receptors but via activation of CCK receptors that may then interfere with opioid actions via postreceptor mechanisms. Key sites for these CCK-opioid interactions are likely to be the spinal terminals of C-fibres. Here, one possibility is that CCK mobilizes calcium from intracellular stores. This will counter the opioid suppression of the rise in internal calcium produced by depolarization, the basis for opioid reductions in transmitter release. Again, CCK only reverses the suppression of the induced rise in $\left[\mathrm{Ca}^{2+}\right]$ i produced by mu but not delta opioid agonists (Wang et al. 1992).

At the same time, there is evidence for another mechanism in the CCK-opioid interaction that involves the endogenous enkephalins acting on the delta opioid receptor. Here, both CCK antagonists and the presence of inflammation (see below, this section) enhance morphine analgesia, an effect that is prevented by delta opioid antagonists. The theory is, then, that CCK inhibits the release of 
enkephalins - removal of this control allows the increased levels of the enkephalins to cause a delta-receptormediated synergy with the mu receptor (Ossipov et al. 1995; Vanderah et al. 1994). Both theories are not mutually exclusive.

In keeping with CCK reducing opioid analgesia, the ability of morphine to inhibit spinal nociceptive processing is enhanced in the presence of selective $\mathrm{CCK}_{\mathrm{B}}$ antagonists, demonstrating physiological antagonism of morphine antinociception by endogenous CCK under conditions of acute nociception. There is now evidence for this interaction in animal models more relevant to clinical situations such as inflammatory and neuropathic pain models (Ossipov et al. 1995; Stanfa et al. 1994).

As discussed earlier, in neuropathies morphine tends to have a reduced effectiveness, whereas after inflammation morphine has enhanced actions. In fact, a few hours after carrageenan inflammation there are mild increases in the potency of delta and kappa opioid effects but marked increases in the effects of morphine (Ossipov et al. 1995; Stanfa et al. 1992). One reason is that due to the novel peripheral action of opioids and systemic dosing, studies will be confounded by this additional site of action. However, spinal morphine is almost 20 times more potent than in normal rats after carrageenan inflammation. The mechanisms for this latter effect must be central and rapidly induced; the increased opioid actions occur within one hour of the inflammation, ruling out receptor upregulation (Stanfa et al. 1992). In this model, exogenous CCK still attenuates the antinociceptive effects of morphine but CCK receptor antagonism no longer produces an enhancement of the antinociceptive effect of morphine. The most likely basis for these results is a decreased availability of CCK within the spinal cord following carrageenan inflammation, either due to a decreased release of CCK or reduced content within the dorsal horn. This reduced functional activity of CCK in inflammation is therefore a major factor in the enhanced potency of spinal morphine seen in these animals (Stanfa \& Dickenson 1993). In exactly the same model there is also an increased alpha-2 inhibitory tone in the spinal cord. Yet in this case, antagonist studies have shown that noradrenergic activity is not a factor in the altered opioid sensitivity, although it may well reduce inflammation-induced nociception (Stanfa \& Dickenson 1994a).

Neuropathic models reveal that CCK plays an entirely opposite role. In nerve damage, increases in CCK systems have been shown to underlie observed reductions in spinal opioid sensitivity. It has been shown that an increase in spinal CCK (likely to be due to novel synthesis of the peptide in primary afferent fibres; see Xu et al. 1993) leads to a reduction in the potency of spinal morphine in a rat model of neuropathic pain following peripheral nerve injury. If the increased CCK is derived from induction of the peptide in the afferents, the interference would seem to be directed at the presynaptic mu receptor. The opioid responsiveness of this model was restored by $\mathrm{CCK}_{\mathrm{B}}$ antagonism. Different pain states may then lead to changes in the levels and synthesis of CCK that can shift opioid sensitivity in either direction (Stanfa et al. 1994). An important point that arises from these studies is that the attenuation of opioid analgesia by CCK is not global but selective for mu but not for delta opioid receptor events. The prediction would be that delta opioids may have efficacy in pain states where morphine is poorly effective due to enhanced CCK levels.

CCK may then be an endogenous "brake" applied to the antinociceptive actions of morphine. In addition to the alterations in CCK induced by different pain states, behavioural studies have suggested that the release of endogenous CCK is even governed by the environment to which an animal is exposed. It is suggested that CCK released in "safe" situations prevents the acute antinociceptive effects of mu agonists and thus reduces the effects of morphine (Wiertelak et al. 1992). Findings such as these may provide a basis to understand how events such as stress and anxiety alter opioid efficacy. Whatever the case, these studies serve to indicate that the release of CCK is not fixed but varies, in both directions, from its normal state according to both external and internal events.

It appears, therefore, that CCK can act to control spinal morphine analgesia. Attenuation of this negative influence leads to augmented spinal opioid controls. This augmentation, along with the novel peripheral actions of opioids in inflammation and enhanced descending controls, could be an adaptation of intrinsic inhibitory systems to balance enhanced nociception during inflammatory states. In contrast to the natural physiological processes of inflammation, the pathological changes in neuropathic pain states result in counterproductive increases in CCK. CCK antagonists currently developed by the pharmaceutical industry may well enhance morphine analgesia in nonpathological pain states and restore morphine analgesia in humans with neuropathic pains. The predicted anxiolytic effects of these antagonists would be a bonus when used as analgesic adjuncts especially in states of chronic pain where anxiety commonly accompanies pain (Stanfa et al. 1994).

5.3. Dynorphin. In inflammatory states there is an increase in the mRNA in the spinal cord for dynorphin and, to a lesser extent, for enkephalin with all the cells increasing dynorphin synthesis having a preceding rise in c-fos, a protooncogene (Dubner \& Ruda 1992). Dynorphin can mimic some of the increases in excitability seen after inflammation, such as the increased nociceptive responses of neurones, while inhibiting others (Knox \& Dickenson 1987). Kappa opioids can functionally antagonize the mu receptor in the spinal cord, potentially contributing to a decreased morphine effectiveness even though opioids are more potent in inflammatory models (Stanfa et al. 1992). Furthermore, increases in spinal dynorphin levels also occur in neuropathic states where opioid actions tend to be reduced. These generally increased dynorphin levels in different physiological pain models, where opioid actions can be increased or decreased depending on the model, make kappa antagonism of morphine unlikely to be of physiological significance. What the functional consequences of the increases in dynorphin mean to the spinal cord is not known (Stanfa \& Dickenson 1994b), and since dynorphin can elicit NMDA-receptor-mediated effects as well as opioid actions (see Dubner \& Ruda 1992), the picture is complex. Figure 1 depicts some of these opioid interactions with spinal circuitry.

\section{Central hypersensitivity}

One of the most important new concepts related to pain is the idea that the ascending and propriospinal pain mes- 


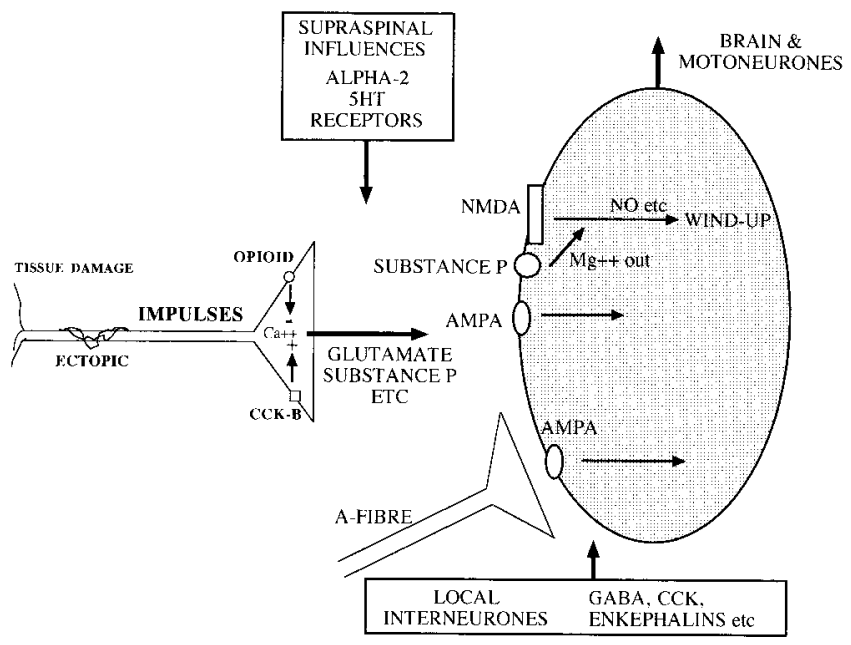

Figure 1. The diagram depicts the interactions between the different pharmacological systems described in section 5 . Activity generated in peripheral sensory nerves releases a number of transmitters into the dorsal horn of the spinal cord. Their receptor actions and interactions, subject to control by local and supraspinal pathways, determine the output from dorsal horn projection neurones. Many of these systems are subject to plasticity.

sages from the dorsal horn are not the same under all circumstances. We are nearing explanations for the extreme aberrations of pain transmission - such as phantom limb pains, hyperalgesias, and allodynias - where the relations between the stimulus and the response are markedly perturbed. The basis for this lack of strict concordance between stimulus and response appears to be the generation of central hypersensitivity (Dickenson 1994b; McMahon et al. 1993; Price et al. 1994b; Woolf 1994; Woolf \& Thompson 1991).

There are two key observations on this subject. First, high-frequency C-fibre stimuli result in a marked and prolonged increase in the flexion withdrawal reflex in rats recorded from motoneurones in spinal animals (Woolf 1983). Thus, noxious stimuli can enhance spinal excitatory events. Second, the repetition of a constant intensity Cfibre stimulus induces the phenomenon of "wind-up," whereby the responses of certain dorsal horn nociceptive neurones suddenly increase markedly (both in terms of magnitude and duration) despite the constant input into the spinal cord (Dickenson 1994b). Volatile general anaesthesia such as with halothane fails to prevent this type of activity, indicating that the treatment of postoperative pain states needs to take into account potential priming events occurring during the operations. The object of this account is to discuss the possible pharmacological substrates underlying these changes.

\subsection{Substrates for central hypersensitivity}

6.1.1. Peptides. Historically Substance P (SP) was the first transmitter to be related to the transmission of pain. SP release can be detected in the spinal cord following highbut not low-intensity peripheral stimulation. The use of antibody microprobes to detect the spatial release of SP has shown that it is essentially restricted to the zones where the C-fibres terminate (Duggan et al. 1988). In addition to substance $\mathrm{P}$, the release of neurokinin A and CGRP following C-fibre activation has been demonstrated. However, when CGRP is present the subsequent release of SP is now extended to cover much of the dorsal horn. The interpretation of this finding is that the degradation of SP is reduced by CGRP binding to the peptidase that also cleaves SP, allowing SP to diffuse in the active form over considerable distances (Schaible et al. 1992). The concept of actions at a distance from the release site, so-called volume transmission, has attracted interest as a basis for nonsynaptic transmitter actions. Events such as these may have relevance to pain as the peptides may diffuse to distant receptors, avoiding both peptidases and spatially restricted inhibitory influences. The induction of inflammation is accompanied by enhanced release of these peptides centrally, which may then contribute to the central hypersensitivity (Dray et al. 1994; Sluka et al. 1992; Todd \& Spike 1993).

The postsynaptic receptors for the neurokinin family of peptides, substance $\mathrm{P}$, and neurokinins $\mathrm{A}$ and $\mathrm{B}$ are now well characterized (Otsuka \& Yoshioka 1993). Cloning and sequencing have been achieved. Currently it is understood that there are three subclasses of tachykinin receptors: the neurokinin 1, -2 , and -3 receptors. Early studies on the role of SP in neuronal events in nociception were bedeviled by poorly selective antagonists and nonspecific effects of the drugs. More recent studies have indicated a role of SP at the NK1 receptor in different types of more prolonged nociceptive transmission, including slow excitatory postsynaptic potentials induced by repetitive C-fibre stimulation and C-fibre-induced reflex facilitation. Similarly, the NK1 receptor antagonist RP67580 has only weak actions on acute responses but produces marked inhibitions of the formalin response of the dorsal horn neurones with equivalent effects on the two phases of the response. These recent studies would indicate that the ability of NK1 receptor antagonists to reduce the activation of dorsal horn neurones depends on the type of stimulation used (Otsuka \& Yoshioka 1993). A consensus is that the conditions for the release of substance $\mathrm{P}$ from the fine afferents include a sufficiently long stimulus at an intensity sufficient to activate C-fibres (Urban et al. 1994). The acute responses of the neurones must therefore include some other transmitter; the evidence implicates glutamate and aspartate. We lack antagonists for the other peptides, but some, such as galanin and neuropeptide $\mathrm{Y}$, are induced in afferents after nerve damage (Todd \& Spike 1993; Urban et al. 1994).

6.1.2. Excitatory amino acids. A large proportion of peripheral sensory fibres including both small and large fibres contain glutamate and aspartate (Battaglia \& Rustioni 1988). In the case of the C-fibres, the coexistence of glutamate with peptides (Battaglia \& Rustioni 1988) makes it highly likely that a noxious stimulus releases both peptides and excitatory amino acids from the afferent nociceptive fibres. Thus in clinical pain states postsynaptic activation of both neurokinin and other peptide receptors together with the receptors for the excitatory amino acids on nociceptive neurones will occur. The development of selective agents for the receptors - the N-methyld-aspartate (NMDA), the metabotropic, and the alphaamino-3-hydroxy-5-methyl-isoxazole (AMPA) receptors has enabled their roles in spinal processing to be studied.

The metabotropic receptor still has an ill-defined role in pain states but may well contribute by acting to enhance 
NMDA and AMPA receptor function via intracellular actions. Use of AMPA receptor antagonists indicate that acute noxious but also innocuous stimuli seem to be transmitted via AMPA receptor activation (Dougherty et al. 1992; Neugebauer et al. 1993). The widespread roles of AMPA receptors in CNS function and the lack of nociceptive selectivity mean that the receptor as a therapeutic target looks doubtful. In contrast, the NMDA receptor has become an increasingly important target site as evidence accumulates for a role of the receptor in the enhancement of spinal processing of painful messages (see Dickenson 1990; 1994b; Price et al. 1994a; 1994b) as well as a target site in many long-term events in the brain (Collingridge \& Singer 1990; Daw et al. 1993). In the spinal cord, the NMDA receptor may play a similar role, especially in more prolonged pain states involving hypersensitivity where functional alterations in central transmission processes may occur.

The complexity of the NMDA receptor-channel is striking. In order to operate it, certain specific conditions need to be met: The release and binding of the coagonists for the receptor, glycine and glutamate, are needed together with a non-NMDA-induced depolarisation to remove the resting magnesium block of the channel (Dickenson 1994b). C-fibre-induced release of excitatory peptides, either in a restricted spatial zone or via volume transmission, may provide the required depolarisation to remove the block, since neurokinin receptor antagonists can reduce NMDAmediated responses in the spinal cord (Urban et al. 1994). For these reasons, the NMDA receptor-channel complex is not a participant in "normal" synaptic transmission. Yet when the correct conditions are achieved, the complex will suddenly become activated and add a powerful depolarising or excitatory drive to transmission of pain in the spinal cord, which then appears to lead to enhanced synaptic transmission or hypersensitivity (Dickenson 1990; 1994a; 1994b; Dubner \& Ruda 1992; McMahon et al. 1993; Neugebauer et al. 1993; Price et al 1994a; 1994b; Woolf \& Thompson 1991). Increased release of afferent peptides in inflammation, for example, could facilitate NMDA transmission by more effective removal of the magnesium block of the receptor channel or by increasing the release of the excitatory amino acids themselves (Kangra \& Randic 1990). It is now well established that wind-up and the reflex hypersensitivity are NMDA receptor mediated. Further experiments with formalin indicate that when inflammation is present, "pathological pain" can be distinguished from the acute phase response where there is no damage on the basis of the sensitivity of only the former to NMDA antagonism. Both the induction and the subsequent maintenance of these responses are dependent on NMDA processes (Haley et al. 1990; Neugebauer et al. 1993; Price et al. 1994b).

However, NMDA receptor activation can also influence inhibitory interneurones in the spinal cord; evidence for this appears from carrageenan inflammation where excessive NMDA receptor activation subsequently induces inhibitory influences (Stanfa et al. 1992). Excessive NMDA activation in the CNS is one mechanism behind excitotoxicity and, as a result, elevated NMDA activation may trigger inhibitory systems as an auto-limiting device to prevent over excitation and even cell death. Possibly, the loss of inhibitions to counter NMDA excitatory mechanisms (Woolf \& Doubell 1994) leads to some of the problems of neuropathic pain. In this regard, NMDA-mediated allodynia can be induced by a blockade of spinal inhibitory tone in normal animals (Yaksh 1989). Furthermore, failure of inhibitions could underlie the transition from acute to chronic pain.

Other approaches have revealed roles of the NMDA receptor in spinal pain processes including ischaemia and neuropathic pain states, where NMDA antagonists have beneficial effects weeks after induction of the injury against the hyperalgesia and spontaneous pain. Thus, there is evidence for an involvement of the NMDA receptor in inflammatory pain, neuropathic pain, allodynia, and ischaemic pain. Not only can wind-up be demonstrated in elegant psychophysical studies in humans (Price et al. 1994a) but, crucially, recent evidence also has shown an NMDA dependency of allodynias and wind-up pains in controlled clinical studies (Eide et al. 1994).

6.1.3. Nitric oxide and arachidonic acid. Central plasticity can also involve a gas. Nitric oxide (NO), a diffusible gas, is produced in response to NMDA receptor activation and thus may mediate some or all of the consequences of NMDA receptor activation in nociception (Meller \& Gebhart 1993). Blockers of nitric oxide synthase (NOS) are effective against inflammatory and neuropathic nociception in animals by spinal actions. There are hints that NO may feed back and enhance the release of the afferent transmitters and as a result set up a positive feedback loop (Sorkin 1993). In addition, an induction of NO in the afferents has been reported after nerve damage. Spinal production of arachidonic acid in response to C-fibre stimulation and NMDA receptor activation may achieve the same end; prevention of this could underlie some of the central analgesic effects of nonsteroidal anti-inflammatory drugs (NSAIDs) (Malmberg \& Yaksh 1992).

The evidence for spinal actions of NSAIDs continues to grow. This growth is based not only on the demonstrations of C-fibre-evoked and, more importantly, NMDA-evoked release of prostanoids, but also on the spinal action of NSAIDs that can be shown by intrathecal administration of these agents. In addition, it has been shown that the hyperalgesia produced by NMDA and substance $\mathrm{P}$ is reduced by spinal NSAIDs (Malmberg \& Yaksh 1992). As there is evidence for prostanoid-, NO-, and NMDAmediated release of glutamate and substance $\mathrm{P}$, it would appear that the production of novel mediators by NMDA receptor activation underlies retrograde messenger control of transmitter release (Sorkin 1993).

There will be problems with NOS blockers in therapy, since $\mathrm{NO}$ is an endothelium-derived relaxing factor and systemic administration may induce analgesia but will be accompanied by severe hypertension. However, it has been demonstrated that neuronal NOS differs from that in the endothelium and thus it may be possible to separate these effects.

\section{Consequences of central hypersensitivity}

The presently characterized systems operate in the spinal cord, which appears to mediate central hypersensitivity, a state where amplification and prolongation of the afferent barrage occurs. The established roles of these different transmitter systems may offer novel targets for therapy. This may be important since wind-up, the hypersensitized 
reflex, and several measures of NMDA-dependent activity in neuropathy and allodynia models can be poorly sensitive to opioids (Dickenson 1994b). As discussed earlier, the reasons may be nociception through channels not controlled by opioids (large fibre-induced allodynias), high levels of excitability (NMDA-mediated amplification), or pathological loss of the opioid receptors.

7.1. Treating opioid poorly responsive pain. The first approach is based on animal studies showing that loss or dysfunction of presynaptic opioid receptors can be overcome by increasing the dose of opioid (Lombard \& Besson 1989; Xu \& Wiesenfeld-Hallin 1991). In addition, in pains where the NMDA receptor is operating and there is reduced opioid sensitivity (as in some of the models for inflammatory and neuropathic pain), this too can be overcome by dose escalation (Chapman \& Dickenson 1992; Yamamoto \& Yaksh 1992). The simple augmentation of the dose of morphine should be first tried, although side effects may confound this tactic. Another approach may be the use of high-efficacy opioids such as alfentanyl or sufentanil, but data is lacking on this point. If opioids cannot produce the desired effects, different pharmacological approaches are possible: In the case of the NMDA receptor there are many experimental drugs that effectively block the receptor, the channel, or associated sites. Some of these are in development as potential drugs, but there clearly is a need for agents to be tested now. In fact, ketamine blocks the channel associated with the NMDA receptor and has current use in the relief of pain. Dextrophan and dextromethorphan are also antagonists at this site and are currently used in humans for their antitussive effects. Both have been shown to reduce wind-up itself (Dickenson et al. 1992) as well as to be effective in the Bennett model of neuropathic pain after spinal application (Mao et al. 1993; Tal \& Bennett 1993) and in humans (Price et al. 1994a). Recently, the anti-Parkinson drug, memantine, has been shown to be an effective NMDA antagonist. All could be used to test the clinical effectiveness of NMDA blockade in opioid poorly responsive pains. However, the NMDA antagonists would be effective only in reducing hyperalgesia, not in abolishing the pain (Dickenson 1994b). These agents may turn out to be especially useful in the allodynias, which are sensitive to NMDA receptor antagonists but not to opioids. In fact, not only is there psychophysical evidence for wind-up pain in humans being mediated by NMDA receptors, based on studies with dextromethorphan (Price et al. 1994a), but there is also evidence from clinical trials showing that ketamine can reduce allodynias, hyperalgesias, and cause pain relief in circumstances where opioids had poor or restricted efficacy (Eide et al. 1994).

One practical application of the poor opioid responsiveness of NMDA-mediated pains is that the coadministration of morphine with low doses of an NMDA antagonist should be beneficial in these pain states. This is indeed the case; furthermore the combination has been shown to synergize in one study (Chapman \& Dickenson 1992) and be additive in another model (Yamamoto \& Yaksh 1992). However, both studies have shown that the additional NMDA antagonism restores the opioid sensitivity of the responses. In addition, spinal local anaesthetics synergize with spinal morphine (Akerman et al. 1988), partly due to the ability of the former to reduce NMDA-mediated activity (Fraser et al. 1992). The spinal release of prostaglandins affords another target, and centrally acting NSAIDs have been shown to reduce persistent inflammatory nociception and the behavioural hyperalgesia produced by spinal substance $\mathrm{P}$ and NMDA. Predictably, NSAIDs will synergize with opioids (Yaksh \& Malmberg 1994).

It has been suggested that once these central hypersensitivity states have been induced, they remain active in the absence of peripheral inputs (Coderre et al. 1990). There is counterevidence from both animal studies and in human pain states where there is clear evidence for central changes that are entirely dependent on peripheral inputs for maintenance (Dickenson \& Sullivan 1987; Gracely et al. 1993). Thus it would seem that the central pain hypersensitivity generators are continually triggered by afferent activity. Consequently, there is a place for peripheral local anaesthetics although the symptoms may well reappear once the block wears off. In addition, there is evidence that systemic local anaesthetics may have selective effects on ectopic foci in a damaged peripheral nerve at doses that do not alter conduction in the nerve (Devor et al. 1992). Finally, as stated earlier, spinal sites of action of local anaesthetics include a reduction in wind-up (Fraser et al. 1992).

In all of these studies on opioid poorly responsive pain, the emphasis has been on mu opioids, especially morphine; yet mu and delta opioids and alpha-2 agonists all have similar effects on wind-up, reducing the initial responses of the cells but with wind-up breaking through the inhibitions as the stimulation continues and restoring the cell responses (Dickenson 1991; 1994a). It is unlikely, therefore, that these three systems would have differential effects on NMDA-receptor-mediated events, making drugs such as clonidine unlikely to be alternatives to morphine, at least with regard to spinal events where the NMDA receptor is implicated. In addition, the negative effects of CCK on analgesia are not only directed against mu opioids but also alpha-2 adrenoceptors. However, it is possible that in cases where presynaptic opioid receptors are reduced, such as in cases of peripheral nerve pathology, alpha-2 receptors may persist at postsynaptic sites and thus provide a therapeutic target. Relatedly, there may well be sympathetic blocking effects of clonidine via systemic routes as well as the spinal route, which will be of importance in sympathetically maintained pains. Yet again, alpha-2 adrenoceptor agonists produce powerful potentiations of opioid analgesia (Dickenson \& Sullivan 1993; Yaksh \& Malmberg 1994).

7.2. Preemptive analgesia. The idea of preemptive analgesia has arisen due to the potential for induction of hypersensitivity, genes, and negative influences on opioid controls in addition to the well-established detrimental effects of the stress and hormonal responses to pain. Thus, treating pain before it arises rather than waiting for it to develop appears to have a rational basis (Woolf 1994). Animal studies lend support to this: several measures of central hypersensitivity are less sensitive to opioids given as a post-treatment as compared to preemptive administration (Chapman et al. 1994). Restricting comments to the use of opioids, there are several reasons why the clinical studies on preemptive analgesia have been either negative or have showed relatively weak benefits (Dahl 1994; McQuay 1994). Most have used postoperative pain measures and invariably operative procedures will induce inflammation.

The key issues in comparing pain relief pre- and post- 
operatively are the exact timing of administration and that baseline opioid sensitivity remains the same (McQuay 1994). With the former, it may be that a pretreatment is the same as an early posttreatment, as they both will preempt late-developing central hypersensitivity. Studies with varied timing of opioids on the formalin response support this idea (Chapman et al. 1994). With opioid sensitivity, it is well established in animal models of carrageenan inflammation and arthritis that not only is novel peripheral opioid receptor mediated analgesia rapidly revealed but also spinal opioid sensitivity is enhanced, just as rapidly (Stanfa et al. 1992; Stein 1994). Thus post-treatment with opioids will impinge upon enhanced opioid systems and comparisons with the effectiveness of pretreatments will be biased. In neuropathic pains, where in general opioid sensitivity is reduced, inhibitions are lost, and central and sympathetic processing is aberrant, the impact of pretreatment is far more obvious.

7.3. The roles of inhibitions. When NMDA-mediated central events leading to hypersensitivity are active in the shorter-term models (formalin and the hypersensitive reflex), there is a reduced sensitivity to opioids; whereas once several hours have elapsed (carrageenan and arthritic inflammation), opioid sensitivity is now found to be increased. In the example of the former models, doseescalation can overcome the reduced opioid sensitivity (Chapman et al. 1994). The reasons for these differences could reside in the unchecked NMDA receptor mediation of activity in the shorter-term models, for example, formalin, prior to the induction of the slower-developing inhibitory changes, overcomes opioid inhibitions. The compensatory increases in spinal opioid sensitivity (via altered CCK) and other inhibitions means that the longer term acute pains respond well to opioids. This is partly due to the enhanced opioid effectiveness per se but also because the increased nonopioid (GABA and alpha-2 adrenoceptor mediated) inhibitory events will reduce the NMDA-driven level of excitability (Castro-Lopes et al. 1994; Stanfa et al. 1994). A good example of the potential controlling influence of inhibitions is seen in the formalin response. NMDA receptor activation in the spinal cord amplifies a low level of C-fibre input (Heapy et al. 1987) to generate the characteristic response. The resultant behavioural and neurophysiological responses to the peripheral injection of formalin last for one hour, but the C-fibre inputs continue for over two hours and the peripheral inflammation for even longer periods (Porro \& Cavazzuti 1993). The central responses must surely be curtailed by inhibitory controls. If the plastic changes are leading to compensatory increased central inhibitions such as these, then preemptive approaches may prevent both this beneficial plasticity as well as the target of central hypersensitivity mechanisms. If this is the case, as the preemptive agent wears off, the pain may return in the absence of compensatory inhibitions and thus with greater intensity.

Whereas the roles of inhibitions have generally received less attention than the excitatory systems, in an animal model of allodynia, NMDA antagonists unlike morphine are effective against tactile-evoked nociception (Yaksh 1989). In this model the NMDA-mediated allodynia is induced by a blockade of spinal GABA or glycine inhibitory tone in normal animals. This has bearing on the previously made point that inhibitions are important in controlling and limiting the extent of NMDA receptor participation in nociceptive processing in the spinal cord. Increasing GABA function by the administration of benzodiazepines reduces NMDA-mediated hyperalgesia after ischaemia (Cartmell \& Mitchell 1993); another intriguing example of this is the enhanced NMDA-mediated nociception seen when spinal glycine inhibitions are blocked in a neuropathic model in the rat (Seltzer et al. 1991). In contrast, GABA upregulation may be an intrinsic compensatory mechanism in longerterm inflammation (Castro-Lopes et al. 1994).

Failure of these and other inhibitory controls conceivably may lead to chronicity of pain. In fact there is strong evidence that the hyperalgesia seen in neuropathic models is as much a consequence of loss of inhibitions and reorganization as excess excitations (Dray et al. 1994; Woolf \& Doubell 1994), which could be due to a destructive loss of inhibitory interneurones - itself exacerbated by pharmacological block of these inhibitory systems (Sugimoto et al 1990).

\section{Developmental aspects}

Examination of events in the neonatal spinal cord are of great relevance to the role of modulatory systems in controlling excitation, since the maturation of the inhibitory systems is slow. Paediatric pain control has to take into account the findings that the development of the nervous system is accompanied by marked changes in many transmitters and receptors over time. A number of studies on the anatomical and functional development of the excitatory and inhibitory pharmacology of the rat and human spinal cords suggest that the rodent provides a good model for investigating clinical questions (Fitzgerald 1991).

Studies using the neonatal spinal cord have shown that all the excitatory and inhibitory receptors covered in this account are functional at day one in the rat; many of these neuropharmacological systems are present even before birth. Full maturation of the endogenous transmitter pathways and connections is much slower (Marti et al. 1987), particularly the local inhibitory and long descending controls. The levels of excitatory transmitters, such as substance $\mathrm{P}$ and excitatory amino acids including glutamate, tend to increase during development. At the same time NK and NMDA receptor location in young animals is far more exuberant than in adults, and their numbers decline with time as the receptors shrink back to assume the discrete adult form (Charlton \& Helke 1986). Dendritic development, interneurones (Bicknell \& Beal 1984), and descending inhibitory controls (Fitzgerald \& Koltzenburg 1986) are slowly developing so that modulation of excitability is delayed. The conclusion from these studies is that the transmission of pain in the spinal cord of the young is likely to be exaggerated compared to the adult as a result of excess early development of excitation and delayed maturity of the intrinsic controlling inhibitory systems.

This difference between the young animal and the adult can be seen once more by using the formalin response, but now in neonatal rats (Guy \& Abbot 1992). In the first week of life, the response to peripheral algogen is dramatic and disruptive to normal behaviour. As development proceeds, response declines - until at about three weeks, when inhibitory systems have matured and response resembles the more discrete adult form. 
Opioids and their three receptors also change with time: the relative arrival of the mu, delta, and kappa receptors differs as does the development of the endogenous opioids. Although the opioid receptor affinities resemble the adult at very early stages, the numbers of receptors decline over time (Attali et al. 1990; Sales et al. 1989). The early ontogeny of the opioid receptors means that there is a substrate present for the production of analgesia by exogenous opioids, whereas the controlling influences of endogenous opioid neuronal systems may only appear later in development.

Thus the systems in the adult that generate spinal hypersensitivity may be more effective in the young nervous system, due to less inhibitory influences as a result of immaturity of these systems. Exogenous activation of opioid receptors allows pain in the young to be controlled by adequate analgesia either with opioids alone or with some of the combinations discussed previously. Because activity-dependent plasticity is important in determining maturation of connectivity in the developing nervous system, it has to be considered that uncontrolled pain in the young may have long-term consequences for the neurobiology of pain. In this respect, fears regarding the consequences of opioid use in young children, which are probably unfounded as suggested by the lack of dramatic effect from early opioid exposure on later function (Bardo et al. 1982), pale into insignificance when compared to the possible permanent alterations in sensory processing that uncontrolled pain in the young could produce.

\section{Conclusions}

Many questions remain to be answered about events in different pain states that can alter, in either direction, the analgesic effects of opioids. As has been discussed, this recently accumulating knowledge of plasticity provides a rational basis for combination therapy (Dickenson 1994b;
Dickenson \& Sullivan 1993; Yaksh \& Malmberg 1994). Examples given here fall into two categories: first, the combination of opioids with other inhibitory agents such as alpha-2 adrenoceptor agonists and, second, dual therapy where the nonopioid acts to reduce excitability or to control interfering systems (CCK and NMDA receptor antagonists, NSAIDs, local anaesthetics). Dual therapy such as this has been shown to result in restoration, additivity, or potentiation of opioid analgesia (Dickenson \& Sullivan 1993; Yaksh \& Malmberg 1994). It is also becoming increasingly clear that particular pain states have different plasticities; despite some similarities (e.g., NMDA mediation of hypersensitivity), inflammatory and neuropathic pains are not only very different from each other but cannot be viewed as uniform syndromes. For example, in neuropathic pains the human and animal studies on opioid sensitivity do not reach a consensus; animal studies suggest both good (Attal et al. 1991; Yamamoto \& Yaksh 1992) and poor opioid sensitivity - and the clinical studies are not totally in agreement on this point either (Arner \& Meyerson 1988; Jadad et al. 1992; Portenoy et al. 1990). Particular monotherapies and combination therapies will likely be appropriate for different states within a pain syndrome (McQuay \& Dickenson 1990). Other key points that need investigation are the following: Does a repeated noxious insult alter the plasticity? Knowledge is building up for the neonate, but what happens in the ageing nervous system? The answers to these and other questions relating to plasticity have important consequences for the clinical treatment of pain.

\section{ACKNOWLEDGMENTS}

The funding of research for the author's laboratory is from the Medical Research Council and The Wellcome Trust. Major contributions to this account, both experimental and conceptual, have come from Louise Stanfa, Vicky Chapman, Ann Sullivan, Alison Reeve, Henry McQuay, Eija Kalso, Maria Fitzgerald, and Jane Haley. Their enthusiasm, dedication, and assistance is gratefully acknowledged. 